\title{
Atomic-scale mechanisms for fluorination-enhanced cycling stability of cation- disordered rocksalt cathodes
}

\author{
Linze $\mathrm{Li}^{1}$, Zhengyan $\mathrm{Lun}^{2}$, Dongchang Chen ${ }^{3}$, Yuan Yue ${ }^{3}$, Wei Tong ${ }^{3}$, Guoying Chen ${ }^{3}$, Gerbrand Ceder ${ }^{4}$ and \\ Chongmin Wang ${ }^{1}$
}

${ }^{1}$ Pacific Northwest National Laboratory, Richland, Washington, United States, ${ }^{2}$ University of California -Berkeley, United States, ${ }^{3}$ Lawrence Berkeley National Laboratory, United States, ${ }^{4}$ University of California Berkeley, United States

Cation-disordered rocksalt (DRX) materials are a new class of high-capacity cathode materials that could not rely on Co or Ni. The current bottle neck for DRX cathodes is that these materials usually do not have good cycling stability and the atomic-level mechanism for the capacity decay in these materials is unclear. A detailed atomistic understanding of the structural evolution in DRX oxides under electrochemical cycling is essential for revealing the atomic-scale mechanisms that underpin the electrochemical performances of DRX cathodes and is thus critical for their commercialization.

Herein, using atomic-resolution scanning transmission electron microscopy (STEM), we examine the structural evolution induced by electrochemical cycling in a model DRX oxide cathode, $\mathrm{Li}_{1.2} \mathrm{Ti}_{0.4} \mathrm{Mn}_{0.4} \mathrm{O}_{2.0}$ (LTMO), and its fluorinated variant, $\mathrm{Li}_{1.2} \mathrm{Ti}_{0.2} \mathrm{Mn}_{0.6} \mathrm{O}_{1.8} \mathrm{~F}_{0.2}$ (LTMOF), and correlate the observed structural evolution to the electrochemical performances of the DRX cathodes. The LTMO cathodes delivered an initial discharge capacity of $191 \mathrm{mAh} \mathrm{g}^{-1}$, and the capacity decayed to $145 \mathrm{mAh} \mathrm{g}^{-1}$ after 50 cycles (Fig. 1a). In contrast, the LTMOF cathode exhibited a lower discharge capacity $\left(151 \mathrm{mAh} \mathrm{g}^{-1}\right)$ in the $1^{\text {st }}$ cycle; however, unexpectedly, the reversible capacity continued to increase as cycling progressed, reaching $227 \mathrm{mAh} \mathrm{g}^{-1}$ after 50 cycles (Fig. 1b). Atomic-scale STEM high-angle annular dark-field (HAADF) image of the pristine LTMO shows that the rocksalt crystalline structure is uniform from the inner bulk to the outer surface (Fig. 2a). After 50 cycles, crystallinity was only preserved in the inner bulk of LTMO, whereas the $\sim 10$-nm-thick surface region has become mostly amorphous and populated with nanosized voids (marked by yellow circles in Fig. 2b). Unlike for LTMO, which exhibited cycling-induced surface degradation, the crystalline structure of LTMOF (Fig. 2c) is maintained from the bulk to surface after cycling (Fig. 2d). Upon cycling a transformation from the rocksalt (Fig. 2e) to a spinel-like structure (Fig. 2f) was observed in the LTMOF particles, as evidenced by the additional spots (marked by white arrows in Fig. 2f) appeared in the diffraction pattern after the cycling. A STEM HAADF image (Fig. 2g) of a nanoscale region in the bulk of the cycled LTMOF and the corresponding filtered image (Fig. 2h) show that the nanosized spinel-like domains are dispersed in the rocksalt matrix. Such structural evolution from the rocksalt to a more spinel-like phase is presumably associated with the gradual capacity increase in LTMOF (Fig. 1b).

In conclusion, we reveal a high level of structural degradation initiated from the particle surface, including amorphization and void formation, in cycled LTMO particles, which can impede Li transport through the surface and should be closely correlated to the capacity fade. This surface degradation is much less prominent in cycled LTMOF particles, indicating the crucial role of fluorination in enhancing the structural stability of DRX cathodes, thereby greatly improving the cycling stability of the cathode. Furthermore, we observe a disordered-to-spinellike structural transformation in the LTMOF bulk. In contrast to the traditional belief that such an irreversible structural change would lead to capacity degradation, we find that this transformation surprisingly results in a capacity increase in LTMOF upon cycling. 
a

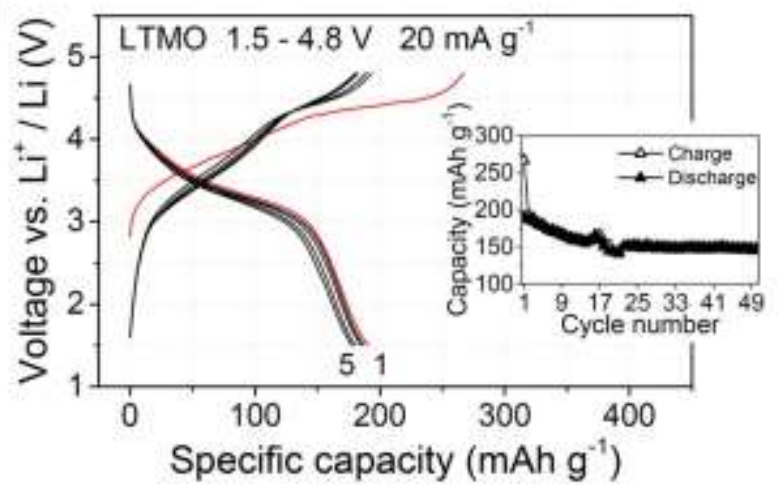

b

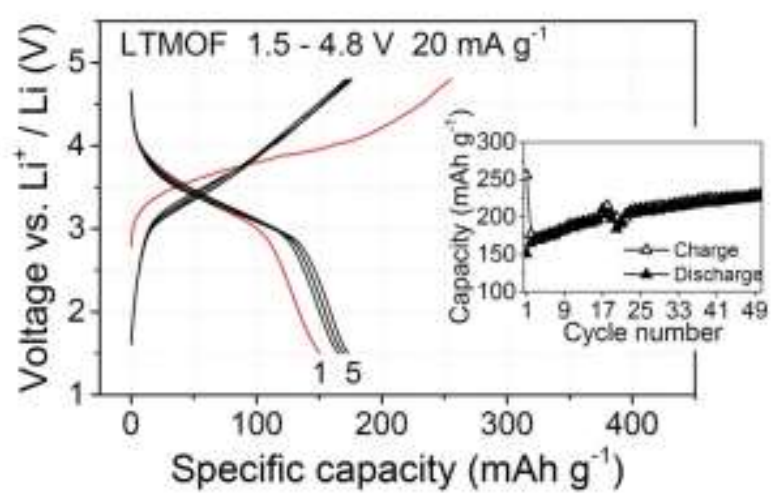

Figure 1. Figure 1. Electrochemical properties of Li1.2Ti0.4Mn0.4O2.0 (LTMO) and Li1.2Ti0.2Mn0.6O1.8F0.2 (LTMOF). (a,b) Charge and discharge voltage profiles for the first 5 cycles and specific capacity as a function of cycle number (inset) for LTMO (a) and LTMOF (b) cathodes in half-cells cycled between 1.5 and $4.8 \mathrm{~V}$ at a current density of $20 \mathrm{~mA} \mathrm{~g}-1$.
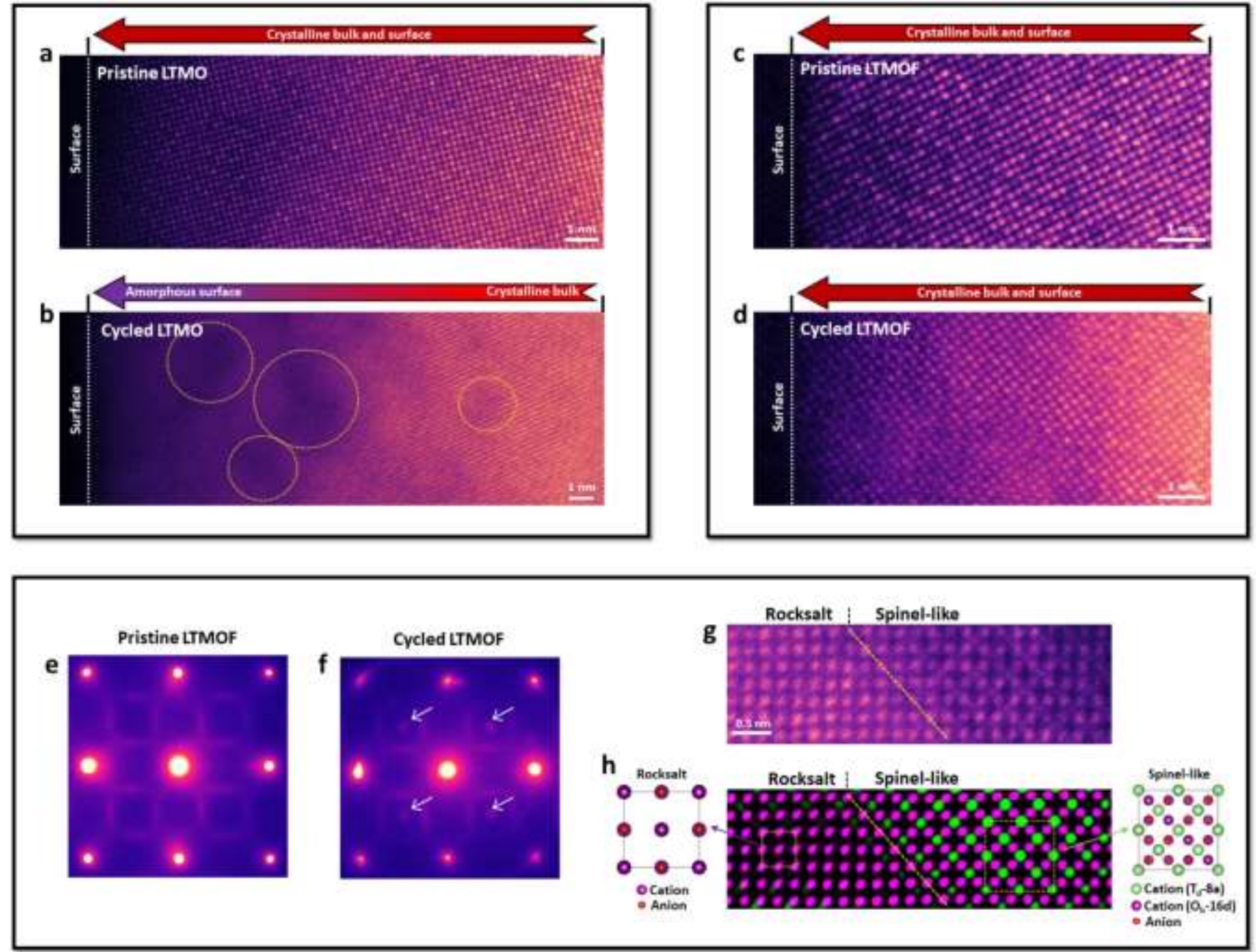

Figure 2. Figure 2. Cycling-induced structural evolution of Li1.2Ti0.4Mn0.4O2.0 (LTMO) and Li1.2Ti0.2Mn0.6O1.8F0.2 (LTMOF). (a,b) Atomic-resolution STEM HAADF images of nanoscale surface regions in LTMO in the pristine state (a) and in the discharged state after 50 cycles (b). The yellow circles in (b) 
mark voids formed below the surface. (c,d) Atomic-resolution STEM HAADF images of nanoscale surface regions in LTMOF in the pristine state (c) and in the discharged state after 50 cycles (d). (e,f) Electron diffraction patterns for pristine LTMOF (e) and discharged LTMOF after 50 cycles (f). The white arrows in (f) mark the 4 additional spots corresponding to the spinel-like lattice. (g) Magnified STEM HAADF image of a boundary across rocksalt and spinel-like structures in cycled LTMOF. (h) Corresponding filtered image of the magnified STEM HAADF image in (g), showing the spatial distribution of rocksalt (purple) and spinel-like (green) structures. 2D atomic models of rocksalt and spinel structures are shown on the left and right sides of the filtered image, respectively.

\section{References}

[1] The author gratefully acknowledges the financial support by the DOE under grant DEAC02-05CH11231 and DE-LC-000L053. 